\title{
A Hierarchical Raster Method for Computing Voronoi Diagrams Based on Quadtrees
}

\author{
Renliang ZHAO ${ }^{1,3}$, Zhilin $\mathrm{LI}^{2}$, Jun CHEN ${ }^{3}$, C.M. Gold ${ }^{2}$ and Yong ZHANG \\ ${ }^{1}$ Department of Cartography and GIS, Central South University, Changsha, China, 410083 \\ \{ zhaorlesina.com $\}$ \\ ${ }^{2}$ Department of Land Surveying and Geo-Informatics \\ The Hong Kong Polytechnic University, Kowloon, Hong Kong \\ $\{$ lszlli@polyu.edu.hk $\}$ \\ ${ }^{3}$ National Geometrics Center of China, \\ No. 1 Baishengcun, Zizhuyuan, Beijing, China, 100044 \\ $\{$ chenjun@nsdi.gov.cn $\}$ \\ ${ }^{4}$ Department of Computer Science and Technology, Tsinghua University ,Beijing, 100084
}

\begin{abstract}
Voronoi diagram is a basic data structure in geometry. It has been increasingly attracting the investigation into diverse applications since it was introduced into GIS field. Most current methods for computing Voronoi diagrams are implemented in vector mode. However, the vector-based methods are good only for points and difficult for complex objects. At the same time, most current raster methods are implemented only in a uniformed-grid raster mode. There is a lack of hierarchical method implemented in a hierarchical space such as quadtrees. In this paper such a hierarchical method is described for computing generalized Voronoi diagrams by means of hierarchical distance transform and hierarchical morphological operators based on the quadtree structure. Three different solutions are described and illustrated with experiments for different applications. Furthermore, the errors caused by this method are analyzed and are reduced by constructing the dynamical hierarchical distance structure elements.
\end{abstract}

\section{Introduction}

A Voronoi diagram is one of fundamental geometric structure, actually describes the spatial influent region for each a generator and each point in the influent region associated with a generator is closer to the generator than the others [1], [14], seen as Fig. 1. Voronoi diagrams have been applied widely in various areas since they were originally used to estimate regional rainfall averages in 1911 [1], [20]. In GIS area, Voronoi diagrams are also taken as one useful tool and have been increasingly attracting the investigation into diverse applications of Voronoi methods [3],[5],[6], [8],[10], [15], [18], [25].

Generally, Voronoi diagrams can be implemented in vector space and also in raster space. But most current methods are vector-based, for example, the classic divide and conquer method, incremental method, sweepline method [1],[9],[15] [20]. 
However, such vector-based methods are good only for discrete spatial points, difficult for complex objects such as line and area objects.

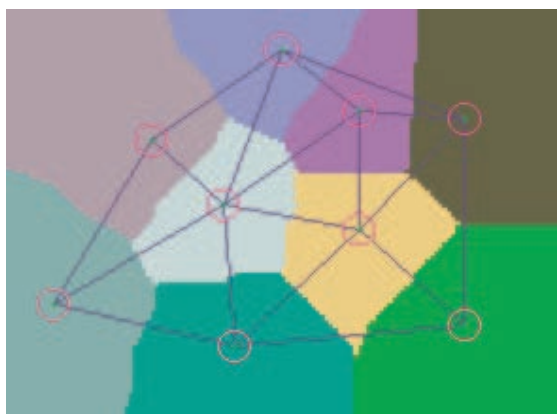

Fig. 1. A Voronoi diagram for points

At the same time, it has been found that Voronoi diagrams can be implemented very well in a raster space. Compared with vector-based methods, raster methods are performed faster and more simply [2],[13],[15],[20]. However, these methods are implemented only in a uniformed grid space. Compared with a uniformed grid structure, a hierarchical structure of space like a quadtree often occupies less space and costs less execution time [23]. In fact, hierarchical methods like that of quadtree have been popular and proved efficient in many areas of GIS including spatial modeling, spatial query, spatial analysis, spatial reasoning and so on[7],[21].

But so far there are a few efforts related to the hierarchical implementation of Voronoi diagrams, despite the fact that the quadtree data structure was used very early in the implementation of Voronoi diagrams. For instance, in Ohya's method, quadtrees are actually only used as an index of buckets to reduce the time of the initial guess of inserted new generator points [17],[19]. However, this method works only for points in a vector space.

In another research closely related to the computation of Voronoi diagrams based on framed-quadtrees, Chen et.al. (1997) adopted a modified quadtree structure to compute Euclidean shortest paths of robotics in raster (or image) space [4]. But the "quadtree structure" there is not the ordinary quadtree, it must be modified into the framed-quadtree whose each leaf node has a border array of square cells with the size of smallest cell before the method can work.

In this paper, a hierarchical method is presented for implementing Voronoi diagrams, directly based on the ordinary quadtree structure as a very good structure representing multi-resolution data or for space saving. In this method, generalized Voronoi diagrams are performed with hierarchical distance transform and mathematical morphological operators in a raster space represented with the standard qudatrees. In the following Section 2, some related operators for the hierarchical computation of quadtrees are introduced. In Section 3 Voronoi diagrams are implemented hierarchically based on these operators, and three solutions are described and illustrated with experiments for different applications. In Section 4 the errors caused by this method are analyzed and a corresponding improvement solution is given by 
means of constructing the dynamical hierarchical distance structure elements. Conclusions are given in Section 5.

\section{Quadtrees and Hierarchical Distance Computation}

As well known, quadtree is one of popular data structure representing spatial data in a variable multi-resolution way in many areas related to space such as GIS, image processing and computer graphics. Quadtree is a hierachical data structure and is based on the successive subdivision of space into four equal-sized quadrants[22], seen as Fig. 2. In such a quadtree, there are three types of nodes: black nodes, grey nodes and white nodes. They represent region of one spatial object, mixed regions of two or more spatial objects and free space respectively. Especially, if a quadtree only records its leaf nodes according to a certain location code, such quadtree is called linear quadtree, while the above quadtree is called regular quadtrees. Different kinds of quadtrees could be suitable for different applications.

The reduction in storage and execution time is derived from the fact that for many images, the number of nodes in their quadtree representation is significantly less than the number of pixels. In terms of the definition of Voronoi diagrams, the formation of Voronoi diagrams is based on the distance. So the most important operation is the computation and propagation of distance in a hierarchical space. This operation can be accomplished with a local method or global method.
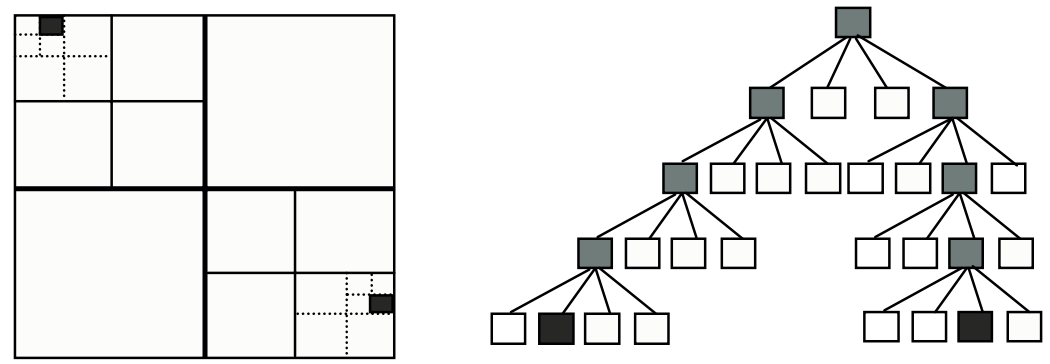

Fig. 2. A regular quadtree

The local method often uses small masks (for instance, the mask consisting of neighbouring pixels with 3 columns and 3 rows) to spread distance and obtain global distance, where the small masks are often associated with definitions of local distance such as city block and Chamfer distance. This is actually the distance transform using small neighbourhoods in image processing [2]. The high efficiency is one of main reasons why this method is popular in image processing. But it will be also costly if the propagation of distance operation covers all pixels of each level. The local method can be straightly generalized to linear quadtrees, shown in Fig. 3(a). The main difference is the distance transform is measured in multiples of the minimum sized quadrant. 
The global method is different the local method. In the global method, the global distance can be directly calculated with an equation of distance in a coordinate system in which each pixel is assigned to a coordinate such as the number of row and column. For instance, the Euclidean distance $D$ between two pixels $P_{l}\left(i_{1}, j_{l}\right)$ and $P_{2}\left(i_{2}, j_{2}\right)$ can be calculated with the following equation.

$$
D\left(P_{1}, P_{2}\right)=\sqrt{\left(i_{2}-i_{1}\right)^{2}+\left(j_{2}-j_{1}\right)^{2}}
$$

The global method can get the distance between any pixels in the whole space more accurately and flexibly than the local method, but the cost will be very high while all of distances between any pixels need.

Therefore, in order to keep the balance between efficiency and precision, it is an alternative choice to integrate the local method and global method, i.e., the distance operation can be accomplished by combining the direct calculation of global distance and the distance transform with small masks. In this mixed method, one can firstly get the global distance between some connected pixels and a spatial object, and then make distance transform outside the spatial object at the beginning of these connected pixels with the global distance. (Seen in Fig. 3(b)) Derived from the above procedure, the efficiency and precision of this integrated method are between the local method and global method for distance calculation.

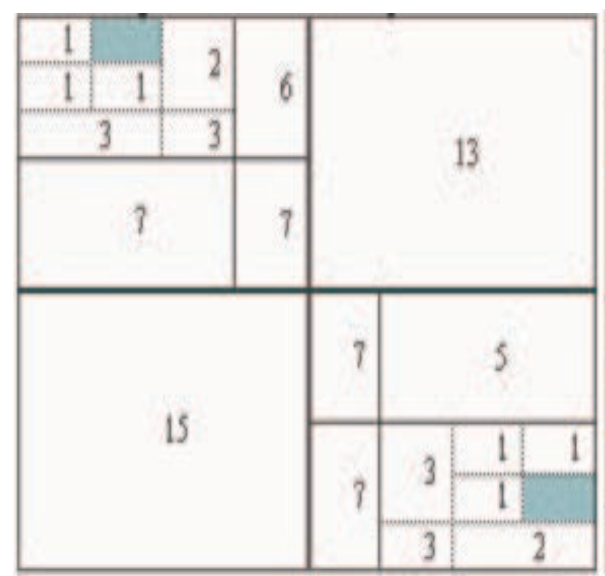

(a)

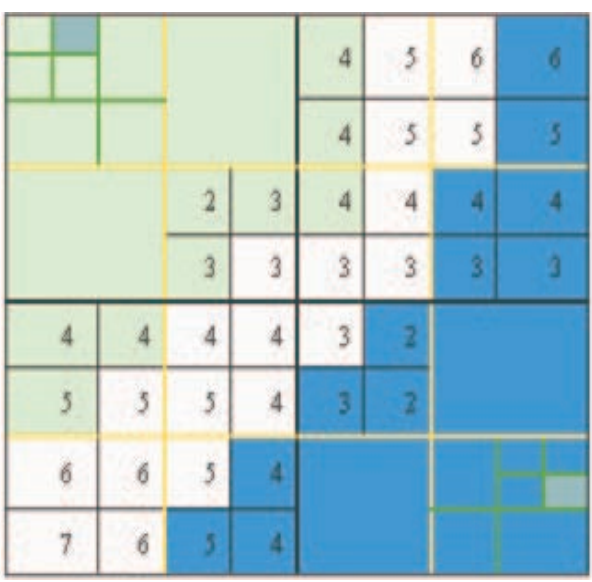

(b)

Fig. 3. (a) The integration of global method and local method in a hierarchical space; (b) Distance transform applied to linear quadtrees

The mixed method can play a better role in the distance calculation of the hierarchical space since it is more flexible than ordinary distance transform. As known, it is derived that some regions of a certain level do not have to continue to be processed in next levels in a hierarchical space. In this case, the flexibility of mixed method makes it possible to omit the distance calculation over the regions unnecessary to be processed. In Fig. 3(b), for instance, it is supposed that the white regions are unnecessary to be continued to process, others are to be continued. With the mixed method, only a part of pixels (with bold lines in Fig. 3(b)) are involved in the global distance 
calculation, the following distance propagation begins with these pixels with the global distance as seen in Fig. 3(b).

In addition to the above approximate method for the distance calculation on linear quadtrees, such distance calculation between free pixels and spatial objects can be also obtained using the dilation operator in mathematical morphology. The dilation operator is one of two basic operators in mathematical morphology, represented as follows [24]:

$$
A \oplus B=\cup_{b \in B} A_{b}
$$

Where $A$ is the original space, and $B$ is the structure element.

The morphological dilation operator has been not only successfully applied to the distance calculation in a uniform grid space [13],[15], but also used for the distance calculation in a hierarchical structure such as the pyramid [16]. Here, it is attempted to implement a new method for the distance calculation using morphological dilation operator in regular quadtrees or linear quadtrees, called hierarchical morphological methods based on quadtrees. The key of a hierarchical dilation operator is substantially made up of the sequence of hierarchical structure elements in quadtrees corresponding to the ordinary structure element. Each hierarchical structure element belongs to a level of quadrees. Fig. 4 shows the structure elements at level 1 and 2 corresponding to 'city block'.
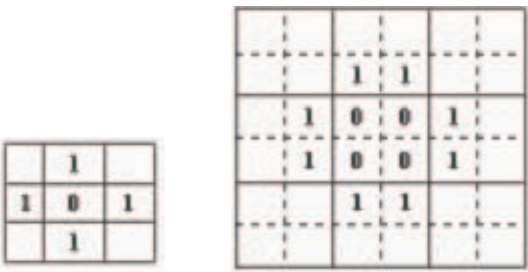

Fig. 4. The structure elements at level 1 and 2 corresponding to 'city block'

\section{Hierarchically Implementing Voronoi Diagrams on Quadtrees}

In raster space, the formation of Voronoi diagrams is substantially viewed as an iterative procedure of subdivision of space. In each iterative step of current ordinary raster methods, each pixel will be determined to belong a certain spatial object according to the distance calculation values. However, it is unnecessary to determine all pixels because possible changed pixels are only those pixels located in boundaries between regions of different spatial objects or between free regions and regions of spatial objects. The application of quadtrees can avoid those redundant operations and finding those possible changed pixels efficiently. The quadtree based hierarchical implementation of Voronoi diagrams is actually also the procedure of continuous subdivision and reconstruction of the quadtree. Due to different ways of hierarchical processing, the hierarchical method for Voronoi diagrams is also implemented in 
different routines. Different routine of the implementation will use different distance calculation.

\subsection{Mixed Distance Calculation Routine}

For regular quadtrees, it is a good way to implement Voronoi diagrams from top to bottom and level by level. In this way, the distance calculation can be accomplished with the mixed method in Section 2. The hierarchical implementation can consist of the following steps.

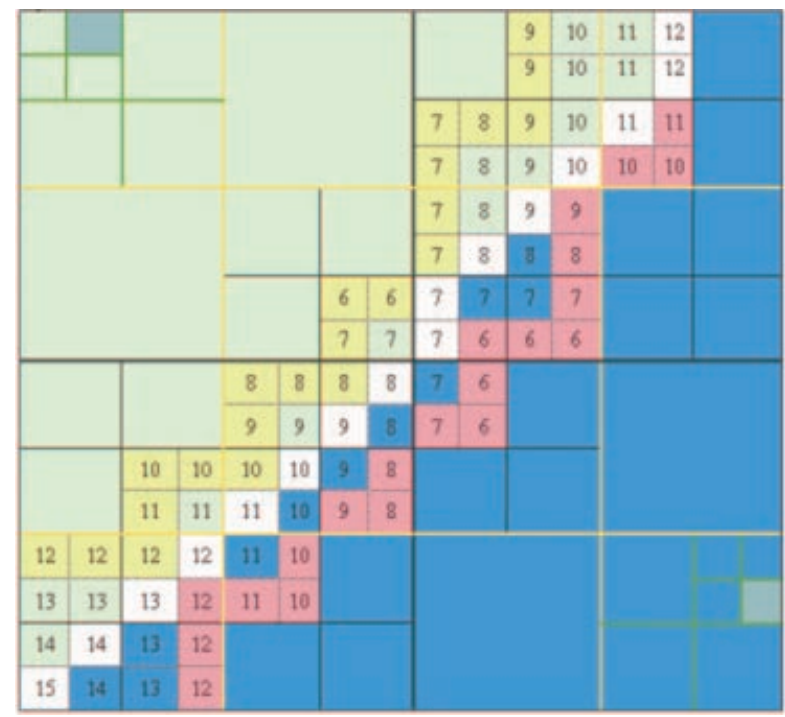

Fig. 5. The hierarchical implementation for Voronoi diagrams using the mixed distance calculation based routine (Total pixels for distance transform $16+40+84=140$, but total pixel will be 256 using non hierarchical methods)

(1) Use global distance calculation method to compute the distance between white nodes and black nodes or grey nodes at the third highest level of the quadtree, then assign those white nodes to the corresponding black nodes or grey nodes

(2) Search for those nodes belonging to each black node or grey node including only one spatial object and not adjacent to other nodes belonging other spatial objects, label them as unused nodes because they are unnecessary to continue to be processed, and label those nodes adjacent to other nodes as boundary nodes;

(3) Subdivide the grey nodes and boundary nodes into less nodes at the next lower level, computing the global distance between less boundary nodes and black nodes or grey nodes including only one spatial objects;

(4) Perform the distance transform using the local method for distance calculation in the regions except nodes labeling 'unused', assign those white nodes to the corresponding black nodes or grey nodes;

(5) Repeat step (2)-(4) until the bottom level. 
Fig. 5 gives an example of the above routine.

\subsection{Linear Quadtrees Routine}

While the raster space is organized into linear quadtrees, it will be very efficient to perform the hierarchical computation of Voronoi diagrams in a way of mixed levels. The feature of this routine is that local distance transform is straightly applied to all levels. Based on the technique, the hierarchical implementation for Voronoi diagrams is described as follows.

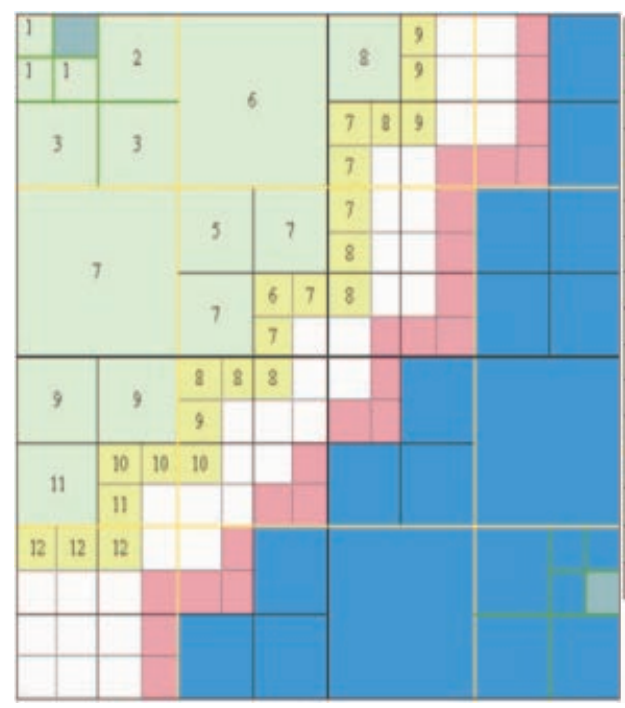

(a)

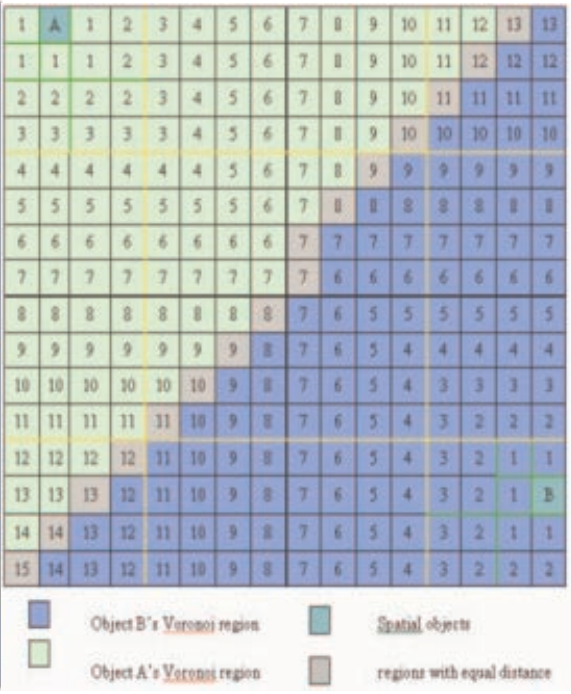

(b)

Fig. 6. (a) The hierarchical implementation for Voronoi diagrams using linear quadtrees based routine, (b) Hierarchical morphological method for Voronoi diagrams

(1) Perform the distance transform on linear quadtrees, make each white nodes associated with a certain spatial object;

(2) Search for those white nodes which belong to each black node and are not adjacent to other white nodes belonging other spatial objects, label them as unused nodes because they are unnecessary to continue to be processed, and label those nodes adjacent to other nodes as boundary nodes;

(3) Subdivide the larger boundary nodes into less nodes at the next lower level,

(4) Perform the distance transform in the regions except nodes labeling 'unused', make each white nodes associated with a certain spatial object;

(5) Repeat (2)-(3) till no white node need subdivision.

An example using this routine is shown in Fig. 6(a). 


\subsection{Morphological Routine}

When original raster data are represented in the uniform grid format, it is necessary to reorganize them into quadtrees firstly for hierarchically computing Voronoi diagrams. At the same time, the hierarchical distance calculation based on morphological method is actually performed at the bottom level of qudtree i.e., the original uniform grids. So in this case, it is suitable for implementing the Voronoi diagrams hierarchically using morphological dilation operator from the each higher level to the bottom level. It can be represented as follows.

(1) Renew to organize the original raster data into a quadtree and construct a sequence of hierarchical structure elements corresponding to the definition of distance in raster space;

(2) For each black node, perform the hierarchical dilation operation stated in the previous section;

(3) Make the distance transform only those pixels dilated by hierarchical operator, assign corresponding spatial object to each pixel of those;

(4) Performing the union operation of quadtrees, merger those dilated pixels and update the current quadtree;

(5) Repeat (2)-(4) till no distance value distance.

Fig. 6(b) illustrates the result of this procedure.

\section{Comparison and Improvement}

The hierarchical implementation of Voronoi diagrams can be realized in three routines. The first routine based on mixed distance calculation method can be suitable for regular quadtrees. The second routine directly using distance transform on linear quadtrees is more suitable for raster data in the format of linear quadtrees. The third routine based on hierarchical morphological operators can be performed in uniformed grid space with the combination of linear quadtrees. More important, the three routines are also different in the efficiency and precision.

From the viewpoint of efficiency, the best routine is the second routine because the distance transform is directly implemented on all levels and it cost less time to get an appropriate distance of all leaf nodes. The time cost time of the third routine is more than the others since it involves many the operation of union quadtrees in each iterative procedure of the implementation of Voronoi diagrams.

In precision, it is derived that the third routine is the best because all operations are actually performed at the bottom level and the result is the same as that of the implementation of non hierarchical methods. In the other two routines, the distance calculation is performed at various levels and causes more error than the third one. However, as pointed out in the literature [15], ordinary distance transforms increase error varying with the growth of distance. This results in larger error of Voronoi diagrams via ordinary distance transforms. In order to improve the precision, an dynamical distance transform hierarchical method is presented here by constructing a set of hierarchical structure of elements close to a circle. 
The key hierarchical structure of elements can be constructed with the method introduced in Section 2, but the difference is that each structure element corresponding to a level here must consist of a set of elements whose combined effect of dilation is required to be a region very close a circle. Applying dynamically these hierarchical dilation operators, the third routine is improved.

\section{Conclusions}

A Voronoi diagram is a geometric subdivision of space that identifies the regions closet to each of its generator sites. Voronoi diagrams have been regarded as an alternative data model and the researches on the applications of Voronoi diagrams have been attracted increasingly in GIS area[5],[8],[9],[11],[12],[15],[25].

The quadtree is very good data structure representing a hierachical or multiscale spatial data. A quadtree based method for the hierarchical computation of Voronoi diagrams in raster space is described in this paper. It can be implemented in three different routines in order to adopt different conditions. Three approaches are different in efficiency and precision. The morphological routine is the best in the aspect of precision, and linear quadtree based routine is more efficient that others in general cases. One can select a suitable routine in his practical needs.

Acknowledgement. The work was substantially supported by a grant from the Research Grants Council of the Hong Kong Special Administrative Region (Project No. PolyU 5048/98E) and partially supported by a grant from the National Natural Science Foundation (No. 40025101).

\section{References}

1. Aurenhammer, Franz, 1991, Voronoi diagrams-A survey of a fundamental geometric data structure. ACM Computing Surveys, 23 (3), 345-405.

2. Borgefors, G., 1986, Distance transformations in digital images. Computer Vision, Graphics and Image Processing, 34, 344-371

3. Chakroun, H.; Benie, G.B; O'Neill, N. T., Desilets, J., 2000, Spatial analysis weighting algorithm using Voronoi diagrams. International Journal of geographical Information Science.14(4), 319-336

4. Chen D. Z., Szczerba R. J. and Uhran, J., 1997, A framed-quadtree approach for determining Euclidean shortest paths in a 2D environment. IEEE Transaction on Robotics and Automation, vol.13, pp668-681

5. Chen, Jun, Li, C., Li, Z. and Gold, C., 2001, A Voronoi-based 9-intersection model for spatial relations. International Journal of geographical Information Science, 15(3): 201220

6. Chen, Jun, Zhao, R.L., and Li., Zhi-Lin, 2000, Describing spatial relations with a Voronoi diagram-based Nine Intersection model. In: SDH'2000, Forer, P., Yeh, A. G. O. and He, J. (eds.), pp4a.4-4a.14 
7. David, M, Lauzon, J. P. and Cebrian, J. A., 1989, A review of qudtree-based strategies for interfacing coverage data with digital elevation models in grid form. Int. J. Geographical Information Systems, 3(1): 3-14.

8 .Edwards ,Geoffrey, 1993, The Voronoi Model and Cultural Space: Applications to the Social Sciences and Humanities In: Spatial information theory : a theoretical basis for GIS : European Conference, COSIT93, Marciana Marina, Elba Island, Italy, September 19-22, Berlin ; New York : Springer-Verlag, Lecture Notes in Computer Science 716, pp202-214

9 .Gahegan, M. and Lee, I., 2000, Data structures and algorithms to support interactive spatial analysis using dynamic Voronoi diagrams, Computers, Environment and Urban Systems, 24: 509-537

10.Gold, C. M., 1994a, a review of the potential applications for Voronoi methods in Geomatics. In: the proceeding of the Canadian Conference of GIS, Ottawa,1647-1652

11.Gold, C.M., 1994b, Advantages of the Voronoi spatial model. In: Frederiksen, P. (ed.). Proceedings, Eurocarto XII; Copenhagen, Denmark, 1994. pp1-10.

12.Gold, C.M.; Remmele, P.R. and Roos, T., 1997, Voronoi methods in GIS. In: Van Kreveld, M., Nievergeld, J., Roos, T. and Widmeyer, P. (eds.), "Algorithmic Foundations of GIS. Lecture Notes in Computer Science No. 1340", Springer-Verlag, Berlin, Germany, pp. 2135.

13.Kotroplulos, C., Pitas, I. and Maglara, M., 1993, Voornoi tessellation and Delauney triangulation using Euclidean disk growing in $\mathrm{Z}^{2}$. IEEE, V29-V32.

14.Lee, D. T. and Drysdale, R.L., 1981, Generalization of Voronoi Diagram in the plane. SIAM Journal of Computing, 10, 73-87.

15.Li, C., Chen, J. and Li, Z. L., 1999, Raster-based methods or the generation of Voronoi diagrams for spatial entities. International Journal of Geographical Information Science, 13 (3), 209-225.

16.Liang, E. and Lin, S.1998, A hierarchical approach to distance calculation using the spread function, International Journal of Geographical Information Science, 12(6), 515-535

17.Marston, R.E.; Shih, J.C. 1995, Modified quaternary tree bucketing for Voronoi diagrams with multi-scale generators Multiresolution Modelling and Analysis in Image Processing and Computer Vision, IEE Colloquium on , 1995 Page(s): 11/1 -11/6

18.Mioc, D.; Anton, F.; Gold, C.M. and Moulin, B., 1998, Spatio-temperal change representation and map updates in a dynamic Voronoi data structure. In: Poiker, T.K. and Chrisman, N.R. (eds.). Proceedings, 8th International Symposium on Spatial Data Handling; Vancouver, BC, 1998. pp. 441-452.

19.Ohya, T., Iri, M. and Murota, 1984, A fast Voronoi diagram with quadternay tree bucketing. Information Processing Letters, Vol. 18, pp. 227-231

20.Okabe, A., Boots, B. and Sugihara, K., 1992, Spatial Tessellations: Concepts and Applications of Voronoi diagrams, Chichester, England, New York, Wiley \& Sons.

21.Papadias, D. and Theodoridis Y., 1997, Spatial relations, minimum bounding rectangles, and spatial data structures. International Journal of Geographical Information Science, 11(2), pp111-138

22.Samet, H., 1982, Neighbor finding techniques for images represented by quadtrees. Computer Graphics and Image Processing, Vol.18, pp.37-57.

23.Samet, H., 1989, Design and analysis of spatial data structures: quadtrees, octress, and other hierarchical methods, Reading MA.

24.Serra, Jean Paul., 1982, Image analysis and mathematical morphology London ; New York : Academic Press.

25.Wright, D.J., and Goodchild., M. F., 1997, Data from the deep: implications for GIS community. International Journal of Geographical Information Science, 11, 523-528 